\title{
Introducing mobile cloud technology into m-health to deliver better care/support in case of emergencies
}

\author{
Hazzaa Naif Alshareef \\ Department of Computer Science, UCC
}

\section{Introduction}

Recent years have seen an increase in the use of mobile devices such as smartphones, tablets, and smart-bands in people's lives. Features offered by these types of devices, such as ease of use and being wireless enabled, allow people to access services that can improve their quality of life. One of the most important aspects of life that can be accessed through mobile devices is health services, whereby users have the ability to track their health status 'on the move', such as by tracking physical activities (e.g., walking and running) and monitoring body status (e.g., heartbeats rate). On the other side, medical professionals and centres can use mobile devices to provide better healthcare services to the public, as doctors, for example, can access patients' records and laboratory results on the move instead of looking at printed charts or files. This reduces the time needed to deliver healthcare to patients. Furthermore, users can communicate with medical professionals in cases of emergency to discuss a medical problem, which could relieve pressure on emergency departments. These practices are known in the health sector as m-health.

However, mobile devices have restricted computational and storage capacity, as well as running on batteries that have limited power. These limitations render mobile devices unable to run the demanding tasks that may be required for accessing health services. Therefore, a solution is needed to allow users to utilize their preferred devices, such as smartphones and tablets, to access or deliver health services with complex and highly computationally heavy tasks being executed, not solely on mobile devices but also on other devices and in different places.

The most suitable solution is to shift heavy computational tasks to remote devices that offer better performance and then return the results to mobile devices without the latter having to do the processing. This is exactly what happens in cloud computing: a health service or application is hosted in the cloud, and mobile devices use this service remotely over the Internet without needing to be concerned about how the service is executed and what resources are needed to get the result. All the user needs to do is request the service and wait for the result. 
Introducing cloud computing technology together with mobile computing technology into the health sector is not a straightforward solution, however, because the integration of these two technologies, known as mobile cloud computing technology, has specific limitations, such as disconnection issues and concerns over privacy and security.

In my research, I examine these kinds of limitations and discuss the possibility of introducing mobile cloud computing technology into the health sector, with the aim of providing a robust, trustworthy system that can deliver healthcare services to mobile users in emergency situations in an easy and reliable way.

\section{Terminology}

\section{M-health}

M-health is an abbreviation of 'mobile health', which means to provide/deliver healthcare services on mobile devices such as smartphones, tablets and wearable devices to minimize shortcomings in the traditional medical framework in an efficient way. Examples of m-health services could be creating electronic medical records (EMR) and making them accessible by mobile devices, establishing a communication link (i.e., video session) between a patient who is facing a medical issue and a doctor who can provide health support to that patient, or retrieving useful information in the case of an emergency, such as the shortest route to the nearest medical centre.

M-health has gained increased attention from researchers in both the medical and information technology (IT) fields for the purpose of reducing the pressure on hospitals, as well as cutting the cost of health services to make them available to everyone. In practice, cloud-based services, featuring both cloud computing and mobile cloud computing technologies, have provided an m-health framework.

\section{Cloud computing (CC)}

Cloud computing (CC) is a new technology that is usually founded on data centres with high levels of computing and storage capacity. CC is now widely used in the computer world because of its desirable features, such as pay-as-you-use schemes, low cost, and great flexibility in implementation. CC has attracted both new developers and the owners of existing systems and, furthermore, represents a new direction and the next generation of the IT industry.

\section{Mobile cloud computing (MCC)}

Mobile cloud computing (MCC) technology is considered a potential solution to deal with mobile device shortcomings, such as the possibility of disconnection and a short battery life. The main differences between this technology and CC technology are that MCC enhances mobility and offers the possibility of building clouds from mobile devices for data storage and computing. MCC also provides functionality for managing data and services 
in a distributed manner that supports several platforms, systems and applications. In the computer field, the term 'MCC' has two definitions. Firstly, a cloud of mobile devices, which means that mobile device resources (storage and computing) are brought together to bring advantages such as access to multimedia and sensor data without the need for large data transfers across computer networks, efficient access to stored data on other devices, and the spread of ownership and maintenance of hardware. Secondly, shifting storage and computing processes from the mobile device space to the CC space, leads to solving mobile device limitations by, for example, saving battery life. The latter definition is widely used in the Information and communications technology (ICT) field and I also consider it in my m-health research project.

\section{Motivating scenarios}

Two different scenarios are presented here that would benefit from a system that can provide health services to the public in emergency situations with the help of MCC technology.

Crowded places

When a large number of people are present in the same place to perform the same activity, such as watching a football match or attending a concert or religious event, there is a high chance that emergency medical situations will occur. For example, in my research, I consider Al-Haj (pilgrimage to Mecca) as such an event, where hundreds of thousands of people are present in the same area, for a certain/limited period of time, performing virtually the same actions. Some issues are related to the medical history of the pilgrims and others can occur because of the environment and the location of this event. Imagine that a person who has a medical history of, for example, heart disease requires healthcare urgently. Owing to the nature of the crowd, reaching such a person with regular emergency services would be difficult or, in some cases, might even be impossible.

However, a doctor or nurse might be located in the crowd, not far from the person in need of help and can, therefore, immediately be available. Alternatively, the person in need could use a mobile device to obtain directions to the nearest medical centre or retrieve useful information that could be vital. Therefore, a system able to link users who are looking for care with those who are able to provide that kind of care is potentially lifesaving.

\section{Residential areas}

Emergency situations can also occur in the home, from cases of people falling downstairs to serious fires that destroy a whole house. Assume now that such an emergency situation has been detected in a home using a modern home-monitoring device. Generally, this will trigger an alert to one of the emergency departments, such as the ambulance and/or fire fighting services. 
Therefore, a multi-notification system is needed: one that automatically searches for doctors or nurses in the vicinity of an emergency situation whilst also providing useful information such as the shortest route to the location. This notification and triggering of an alert at the relevant emergency centre would happen simultaneously.

\section{My research}

As mentioned above, mobile devices allow people to have access to the Internet on the move and mobile cloud computing allows the deployment of services that are accessible over the Internet by mobile users. Therefore, introducing these technologies would improve healthcare applications and benefit a variety of users (including patients and healthcare providers).

The research aims to enhance the delivery of healthcare information (or advice) and expand the point-of-care concept to everybody, anywhere, when they are in distress. This means mobilizing care for people in emergency situations wherever these occur, as well as notifying health providers and establishing communication links (if needed) between all the parties concerned for delivering health services.

Thus, the research focuses on the design and implementation of a mobile cloud healthcare system that delivers at the point of care in order to provide a robust and reliable mobile health system that can deliver fast and low-cost care to the public when experiencing health emergencies. Figure 1 shows an overview of the system model.

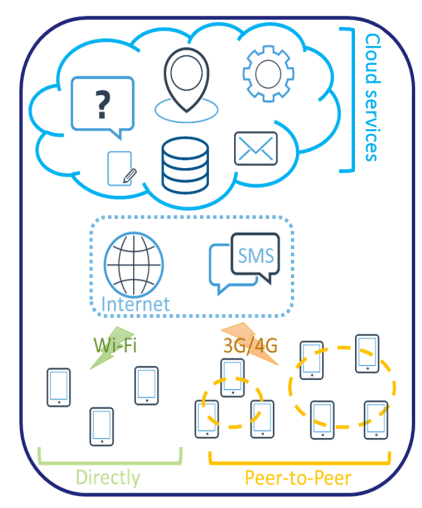

Figure 1: System Model Overview. Source: Author

To achieve this goal, I am working on two main areas: infrastructure and services. In the first area, I enhance the connectivity aspects of the system by allowing mobile users to create/join self-organized peer-to-peer networks. This kind of network is then managed/monitored by the cloud and offers its users the ability to connect to the cloud to seek care, either directly or via a neighbour who has a better connection to the cloud. It also allows the members of such a network to share resources or data locally without the need to use a carrier/caller network (3G/4G) with its associated costs. 
Similarly, the cloud can reach users directly or via a neighbouring link. This method will increase the system availability, as well as reducing or minimizing the risk of disconnection, which is one of the shortcomings of deploying mobile cloud computing technology.

Moving to the second area, which is creating services that are hosted in the cloud and can be accessed by mobile users, these services can operate together to deliver better healthcare services to users whenever they are needed. Location, medical state, and the connectivity status of users are tracked by the cloud for the purpose of providing the best possible outcomes.

An example of such a service could be sending a request to the cloud to retrieve medical advice that could help in delivering a healthcare service to a user who is facing an emergency situation on the motorway. Another service could be to allow users to find doctors or nurses who might only be a few steps away from them in order to discuss a medical concern. A service could also link a monitoring device (such as a body sensor) whereby if an emergency case is detected a request for help would be sent automatically without the need to involve the user in the operation.

However, designing and implementing such a system will generate a number of challenges. These challenges can be divided into three main groups, which are presented below.

Challenges related to technologies in use: As stated previously, cloud computing technology was introduced to offer better outcomes (such as high performance and high availability). As is known, cloud computing still suffers from some shortcomings, such as issues related to security, privacy, reliability, and availability. In my proposed system, security and privacy are the most important issues because the technology is required to deal with people's medical data, which are very sensitive and have to be protected and secured. Therefore, the system has to provide a strong security mechanism that ensures that the content of medical data is stored securely in the cloud using high-level protection strategies, such as data encryption. The system also needs to ensure that both cloud services and stored data can be accessed by the right people to minimize the possibility of attack. This requires ensuring access to the system using a high-level authentication technique, such as a one-time-password or SMS-authentication scheme or one of the biometric authentication methods that offer a high degree of protection.

Challenges related to users who participate in the system: In addition to users who are seeking health services and want to ensure their sensitive medical data are protected and secured, there is another kind of user: those who provide health services to the public and have specific requirements to help them deliver care or support to people in emergency situations. These requirements include ease of use, fast response, and high availability. It is also understood that providing health support to the public in an out-off-hospital way will put more pressure on healthcare professionals. Using the concept of volunteering is a key solution to this issue, whereby a medical 
professional, or anyone who is qualified, authorized and able to deliver healthcare to the public, has the choice of participating or not. The system also has to allow them to change their status if required; for example, from "available" to "offline" or to "dealing with a case". Finally, the system has to have a form of management that ensures all participating users are treated equally in the case of redirecting medical requests. Carrying out a survey to discover medical professionals' views of using such a system could be useful to my research.

Challenges related to the collected data: To provide a healthcare service to someone, some data have to be collected, such as current location, medical history (including body monitoring data, e.g., heart rate and blood pressure), and personal information (e.g., name and age). This raises ethical issues regarding how this type of information will be used and who will access the data. Collecting this type of information is critical for the proposed system to provide proper help to that person. For example, the system has to be aware of any allergies or special medical requirements (e.g., heart-related issues or asthma) before redirecting the case to an available medical professional. Another example is related to tracking technology, as the system has to identify the current location of the person seeking help in order to connect him/her to medical staff who are in the vicinity. Furthermore, the system has to track medical professionals for the purpose of redirecting medical emergency cases that are close to their location. Therefore, collecting this type of data is very important and might be life-saving. However, the system has to provide a high level of confidence to users that their data will be used only for medical purposes and they have the right to accept or reject sharing this type of information with other medical parties, such as hospitals or insurance companies.

With regard to the design process, the first step was to define the research problems and then review similar projects and related research. Then, I formulated hypotheses to start the system design, during which stage I designed my system taking into account its suitability for implementation in real-world scenarios. Now, I am testing the design in a real environment to check the viability of the system. I am also analysing the results collected during the previous step to make recommendations that should help in dealing with the problems that were defined in the first step. I may go from this step to the design stage for the purpose of making any modifications that are required to obtain clearer results or correct any issues in the design part. Figure 2 shows the design process.

To summarize, I hope that future healthcare systems can benefit of my research in enabling the delivery of better care to the public as well as reducing pressure on medical centres.

Hazzaa N. Alshareef is a PhD student in the Mobile and Internet Systems Laboratory (MISL) in the Department of Computer Science at University College Cork (UCC) under the kind supervision of Dr Dan Grigoras. His research is funded by the Saudi Electronic University in 


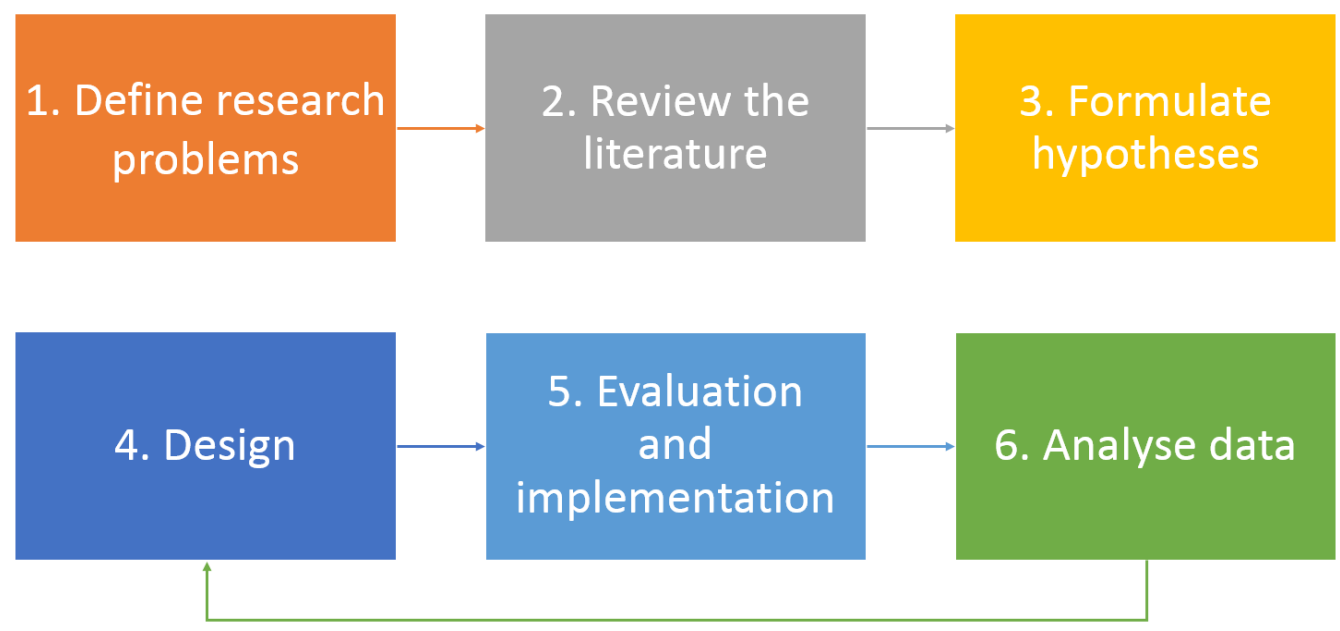

Figure 2: Design progress chart. Source: Author

Saudi Arabia. He is forever indebted to his supervisor, Dan Grigoras, his MISL colleagues and his family, Norah, Naif and Najat, for their help and support during his journey. 